\title{
mTORC1 stimulates phosphatidylcholine synthesis to promote triglyceride secretion
}

\author{
William J. Quinn III, ${ }^{1}$ Min Wan, ${ }^{1}$ Swapnil V. Shewale, ${ }^{2}$ Rebecca Gelfer, ${ }^{1}$ Daniel J. Rader, ${ }^{2}$ Morris J. Birnbaum,, \\ and Paul M. Titchenell ${ }^{1,4}$ \\ IInstitute for Diabetes, Obesity, and Metabolism, and 2'Cardiovascular Institute, Perelman School of Medicine at the University of Pennsylvania, Philadelphia, Pennsylvania, USA. ${ }^{3}$ Internal Medicine, \\ Pfizer Inc., Cambridge, Massachusetts, USA. ${ }^{4}$ Department of Physiology Perelman School of Medicine at the University of Pennsylvania, Philadelphia, Pennsylvania, USA.
}

\begin{abstract}
Liver triacylglycerol (TAG) synthesis and secretion are closely linked to nutrient availability. After a meal, hepatic TAC formation from fatty acids is decreased, largely due to a reduction in circulating free fatty acids (FFA). Despite the postprandial decrease in FFA-driven esterification and oxidation, VLDL-TAG secretion is maintained to support peripheral lipid delivery and metabolism. The regulatory mechanisms underlying the postprandial control of VLDL-TAG secretion remain unclear. Here, we demonstrated that the mTOR complex 1 (mTORC1) is essential for this sustained VLDL-TAG secretion and lipid homeostasis. In murine models, the absence of hepatic mTORC1 reduced circulating TAG, despite hepatosteatosis, while activation of mTORC1 depleted liver TAC stores. Additionally, mTORC1 promoted TAG secretion by regulating phosphocholine cytidylyltransferase $\alpha$ (CCT $\alpha$ ), the rate-limiting enzyme involved in the synthesis of phosphatidylcholine (PC). Increasing PC synthesis in mice lacking mTORC1 rescued hepatosteatosis and restored TAC secretion. These data identify mTORC1 as a major regulator of phospholipid biosynthesis and subsequent VLDL-TAG secretion, leading to increased postprandial TAG secretion.
\end{abstract}

\section{Introduction}

In response to periods of fasting and nutrient abundance, the liver synthesizes, stores, and secretes triacylglycerol (TAG) to maintain organismal health. During starvation, hepatic TAG content is elevated, largely due to the influx of circulating free fatty acids (FFAs). In response to a meal, hepatic TAG levels are reduced in part by a reduction in FFAs and the maintenance of VLDL-TAG secretion. (1-3). The stimulation of VLDL-TAG export from the liver facilitates systemic lipid transport to extrahepatic tissues. Importantly, VLDL-TAG secretion is central to the regulation of hepatic and circulating lipid levels; thus, increases in VLDL-TAG contribute to hyperlipidemia and metabolic disease $(4,5)$.

Critical to the physiological regulation of hepatic lipid export is the anabolic hormone insulin, which regulates VLDL-TAG metabolism via multiple pathways, including both transcriptional and translational mechanisms (6). Chronic insulin infusion increases VLDL-TAG production in spite of significant reductions in FFA, suggesting that insulin promotes VLDL secretion (7). Moreover, genetic disruption of hepatic insulin signaling in mice reduces triglyceride secretion, lowers serum TAG levels, and reduces atherogenesis in animal models (8-10). On the other hand, in isolated hepatocytes, insulin treatment inhibits VLDL-TAG and apolipoprotein B secretion $(11,12)$. Therefore, there are conflicting reports on the role of hepatic insulin signaling in the control

Conflict of interest: M. Wan is currently an employee of Agios Pharmaceuticals. M.J. Birnbaum is an employee of Pfizer Inc. P.M. Titchenell receives research funding from Pfizer Inc.

Submitted: June 30, 2017; Accepted: September 5, 2017

Reference information: J Clin Invest. 2017;127(11):4207-4215.

https://doi.org/10.1172/JCI96036. of VLDL secretion. In the studies described below, we explore the signaling pathways downstream of hepatic insulin action to define the role of critical downstream insulin signaling mediators in the regulation of VLDL secretion and lipid homeostasis in vivo.

During feeding, insulin and amino acids activate the mTOR complex 1 (mTORC1), a major downstream mediator of insulin action on cellular anabolic processes, including the synthesis of protein, lipids, and nucleotides (13). mTORC1 signaling is required, but not sufficient, for postprandial SREBP1c activation and de novo lipogenesis (14-16). The inactivation of mTORC1 upon fasting is critical to the induction of ketogenesis (17). Thus, mTORC1 has been implicated in both the breakdown and synthesis of hepatic fatty acids by insulin. Yet its role in the synthesis and export of complex neutral lipids and phospholipids remains largely unexplored. Here, we test the hypothesis that activation of mTORC1 is an essential regulator of hepatic TAG secretion in vivo. Toward this end, we identify a mechanism by which, through the control of phosphatidylcholine (PC) biosynthesis, mTORC1 is a major regulator of hepatic TAG secretion in vivo.

\section{Results}

mTORC1 activity regulates postprandial liver TAG content. To understand the contribution of mTORC1 to levels of hepatic triglyceride during the transition from fasted to feeding, we injected fasted mice with the mTORC1 inhibitor rapamycin prior to refeeding. Rapamycin completely blocked the reduction in hepatic TAG levels upon refeeding (Figure 1A). To establish whether this effect was due to inhibition of hepatic mTORC1, we performed liver-specific ablation of the expression of raptor (L-Raptor-KO) or TSC1 (L-TSC-KO) in vivo, thus constitutively inhibiting or activating mTORC1, respectively. Adenoassociated virus expressing CRE recombinase under 
A

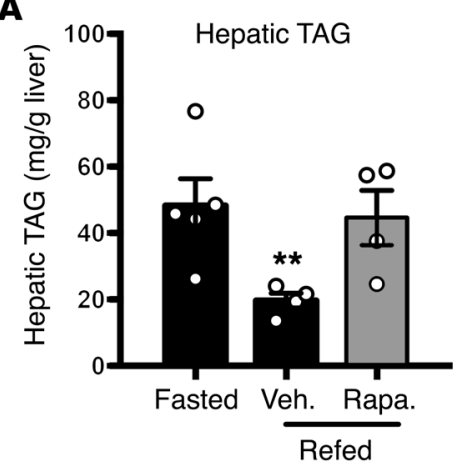

C

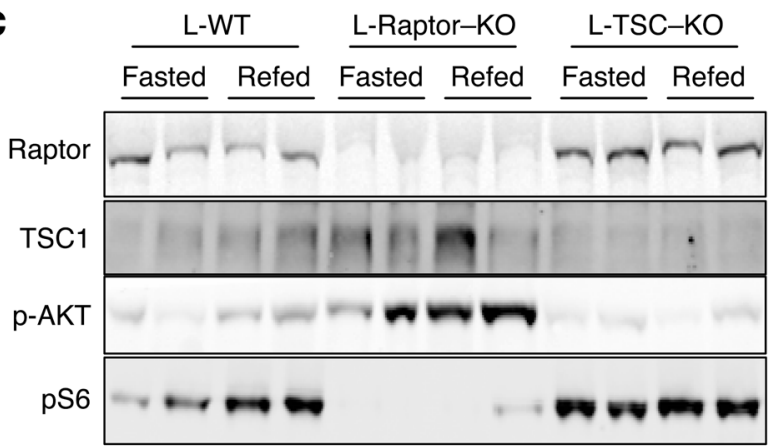

E

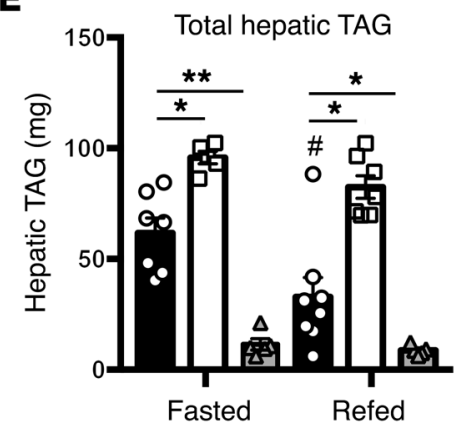

$\mathbf{F}$

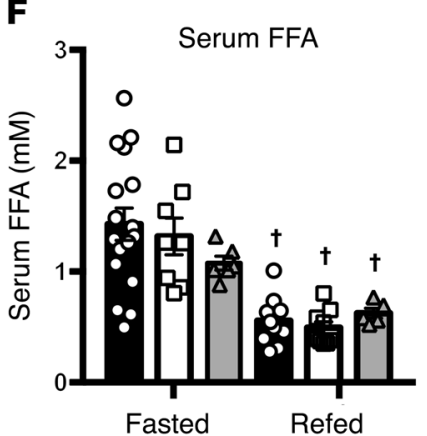

B

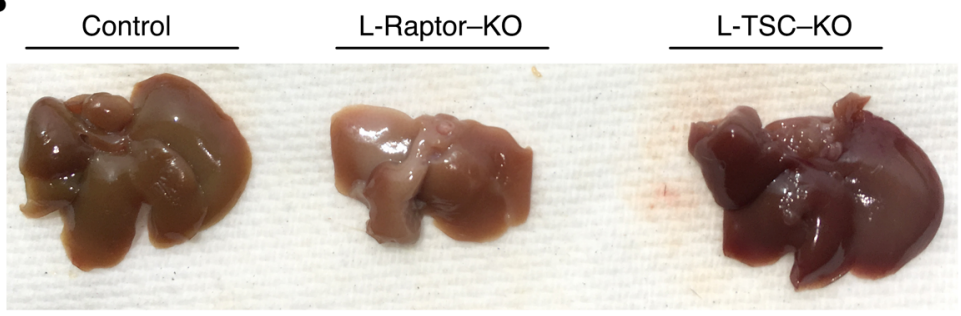

D

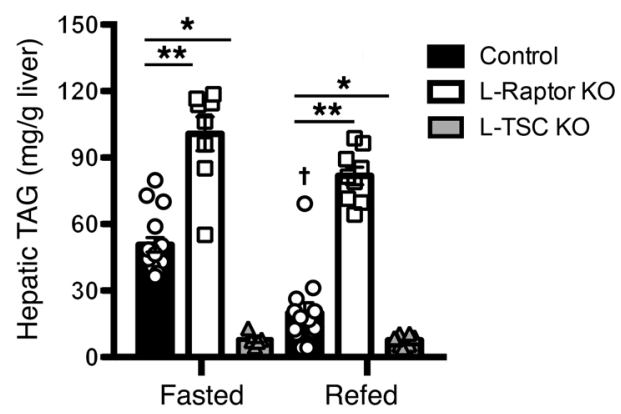

G

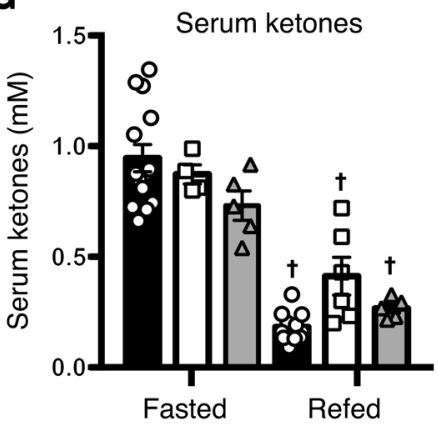

Figure 1. mTORC1 activity is both required and sufficient to induce hepatic steatosis. Fasted 8- to 12-week-old C57BL/6 mice were injected with 20 mg/ kg of rapamycin (A) liver TAG; $n=4$. Veh., vehicle; Rapa, rapamycin. (B-C) Six- to ten-week-old Raptor fl/fl and Tsc $7^{f l / f l}$ animals were injected with either AAVGFP (control, black) or AAV-CRE (L-Raptor-KO, white; L-TSC-KO, gray) for 2 weeks prior to sacrifice. Cohorts were either fasted for 18 hours or fasted and refed for 4 hours. (B) Livers from mice of the indicated genotypes. (C) Immunoblot for indicated proteins. (D) Hepatic TAG was measured. (E) Total hepatic TAG normalized to liver. (F) Serum FFAs. (G) Serum ketones. $n=5-12$ per group. ${ }^{*} P<0.05$; ${ }^{*} P<0.01$ vs. control. ${ }^{*} P<0.05$ vs. fasted (when comparing refed vs. fasting). ${ }^{\dagger} P<0.01$ vs. fasted (when comparing refed vs. fasting). Two-way ANOVA.

the control of the liver-specific thyroxine binding globulin (TBG) promoter was injected into mice homozygous for floxed alleles of either Raptor or Tsc1. L-floxed mice injected with an adenoassociated virus expressing GFP served as a control. Livers from L-RaptorKO mice appeared pale and smaller than those of both control and L-TSC-KO (Figure 1B). As expected, feeding failed to increase the phosphorylation of S6 in L-Raptor-KO, while L-TSC-KO mice displayed constitutive phosphorylation of S6 (Figure 1C). See complete unedited blots in the Supplemental material. In addition, L-RaptorKO mice showed an enhanced phosphorylation of Akt upon feeding due to the lack of negative feedback by mTORC1. Under fasting conditions, L-Raptor-KO mice had increased hepatic TAG, which tended to be reduced following refeeding, but did not reach statistical significance (Figure 1D). Conversely, constitutive activation of
mTORC1 (L-TSC-KO) led to significantly reduced liver TAG during both fasting and the prandial state (Figure 1D). In addition, L-Raptor-KO mice exhibited an increase in total hepatic TAG despite a reduction in liver size, while L-TSC-KO mice had significantly reduced TAG in liver (Figure 1E). Moreover, serum FFAs and ketone levels were suppressed appropriately following refeeding, despite the increase in hepatic TAG in the L-Raptor-KO (Figure 1, F and G). These data are consistent with the hypothesis that mTORC1 activity in the liver is a critical cell-autonomous regulator of neutral lipid in liver independent of changes in circulating FFAs levels. Additionally, the inverse correlation between mTORC1 activity and liver fat is seemingly at odds with the well-known function of mTORC1 to promote fatty acid synthesis, suggesting mTORC1 controls hepatic lipid homeostasis via multiple pathways $(15,16)$ 
A
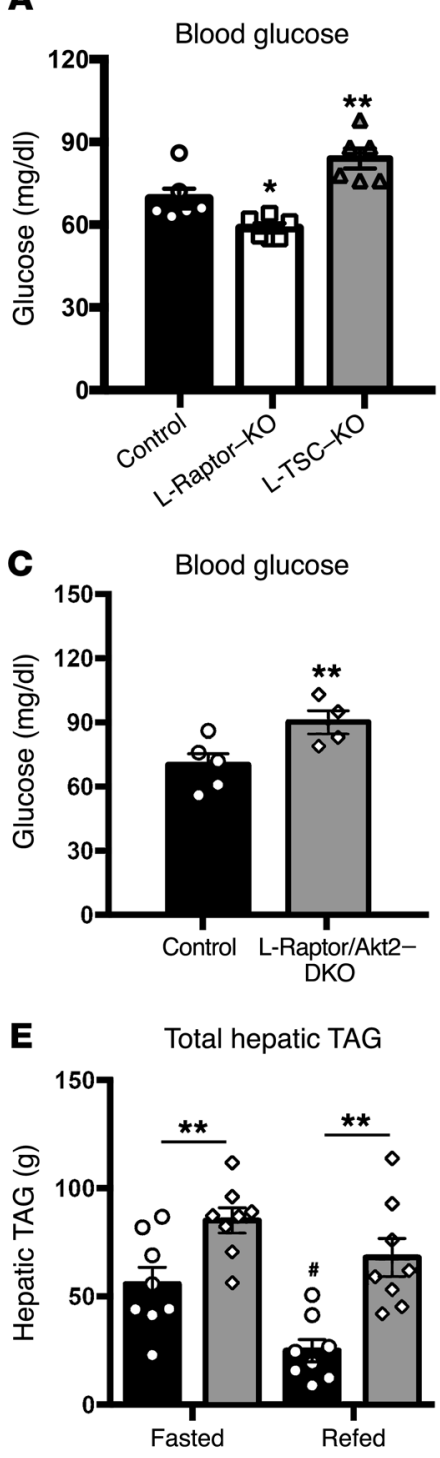

B

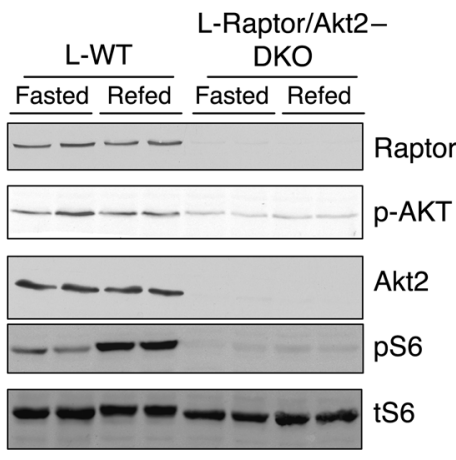

Figure 2. Loss of mTORC1 increases hepatic TAG independently of Alkt. Six- to ten-week-old Raptor $^{f l / f l}$ and $T s c f^{f l / f l}$ animals were injected with either AAV-GFP (control, black) or AAV-CRE (L-Raptor-KO, white; L-TSC-KO, gray) for 2 weeks prior to sacrifice. (A) Fasting blood glucose. $n=5$. (B-G) Six- to ten-week-old Akt2/Raptor fl/fl animals were injected with either AAV-GFP (control, black) or AAV-CRE (L-Akt2/Raptor-DKO, gray) for 2 weeks prior to sacrifice. (C) Fasting blood glucose. (D) Hepatic TAG was measured. (E) Total Hepatic TAG normalized to liver. (F) Serum FFAs. (G) Serum ketones. $n=4-8$. ${ }^{*} P<0.05$; ${ }^{*} P<0.01$ vs. control. ${ }^{*} P<0.05$ vs. fasted (when comparing refed vs. fasting). ${ }^{\dagger} P<0.01 \mathrm{vs}$. fasted (when comparing refed vs. fasting). Two-way ANOVA.

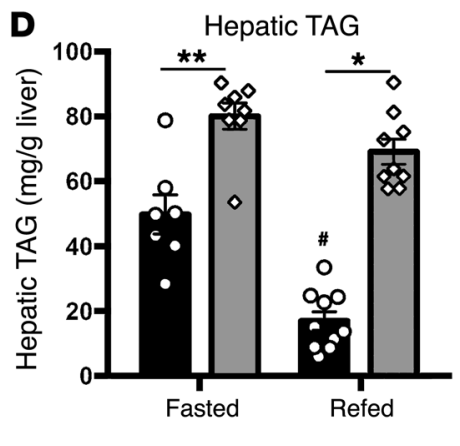

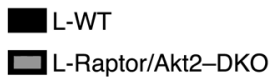

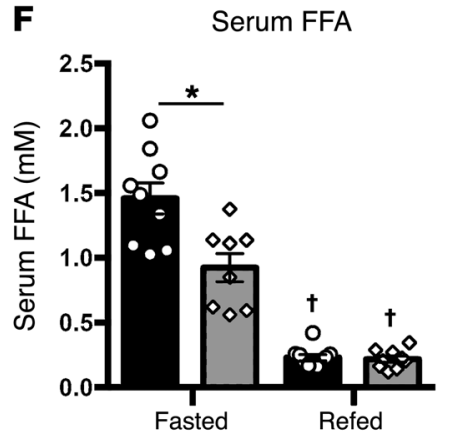

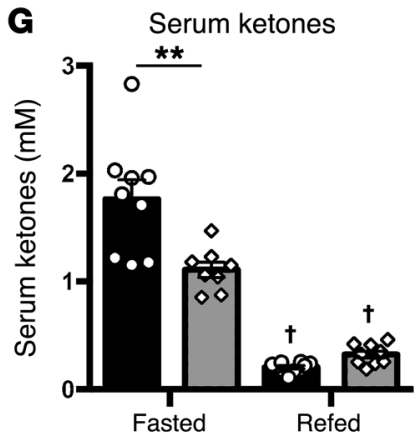

The increase in liver TAG in L-Raptor-KO mice cannot be explained by elevated Akt activity. Akt signaling is both required and sufficient to increase hepatic TAG levels (18). Activation of Akt in liver is regulated in part by a negative feedback loop downstream of mTORC1 (19). In agreement with this model, phosphorylation of Akt was enhanced during both fasting and refeeding in L-Raptor-KO while constitutive activation of $\mathrm{mTORC} 1$ in the L-TSC-KO reduced phosphorylated Akt (p-Akt) (Figure 1C). Consistent with activation of Akt in the fasted state, L-Raptor-KO displayed a significant reduction while L-TSC-KO had elevated fasting blood glucose (Figure 2A). To determine whether the accumulation of hepatic TAG in the absence of mTORC1 was due to activation of Akt, we generated mice lacking both Akt2 (the major Akt isoform expressed in liver) and Raptor (L-Raptor/Akt2 DKO) in liver, blocking the activation of p-Akt caused by loss of Raptor (Figure 2B). See complete unedited blots in the Supplemental material. L-Raptor/Akt2-DKO mice had elevated fasting blood glucose, consistent with the idea that enhanced Akt signaling was responsible for the reduction of fasting blood glucose in L-Raptor-KO (Fig- ure $2 \mathrm{C}$ ). In contrast, despite significant reduction in Akt signaling, L-Raptor/Akt2-DKO mice nonetheless maintained increased hepatic TAG in both fasting and refed conditions (Figure 2, D and E). Additionally, refeeding suppressed FFAs and ketones normally in L-Raptor/Akt2-DKO (Figure 2, F and G). These data support the hypothesis that mTORC1 controls the postprandial reduction in hepatic lipid accumulation downstream of Akt.

mTORC1 regulates VLDL TAG secretion. Hepatic TAG secretion supplies lipid to peripheral tissues, while defects in lipid export can lead to hepatosteatosis $(20,21)$. L-Raptor-KO had significant reductions in serum TAG, suggesting that decreased TAG secretion was responsible for the accumulation of neutral lipid in liver (Figure 3A). Serum fractionation studies revealed a dramatic reduction in VLDL-TAG in the L-Raptor-KO (Figure 3B). In addition, mice with constitutive activation of mTORC1 (L-TSC-KO) displayed significantly more TAG in the VLDL fraction, suggesting that hepatic mTORC1 controlled TAG secretion (Figure 3B). To directly ascertain rates of triglyceride secretion in vivo, we injected fasted control, L-Raptor-KO, and L-TSC-KO mice with polox- 

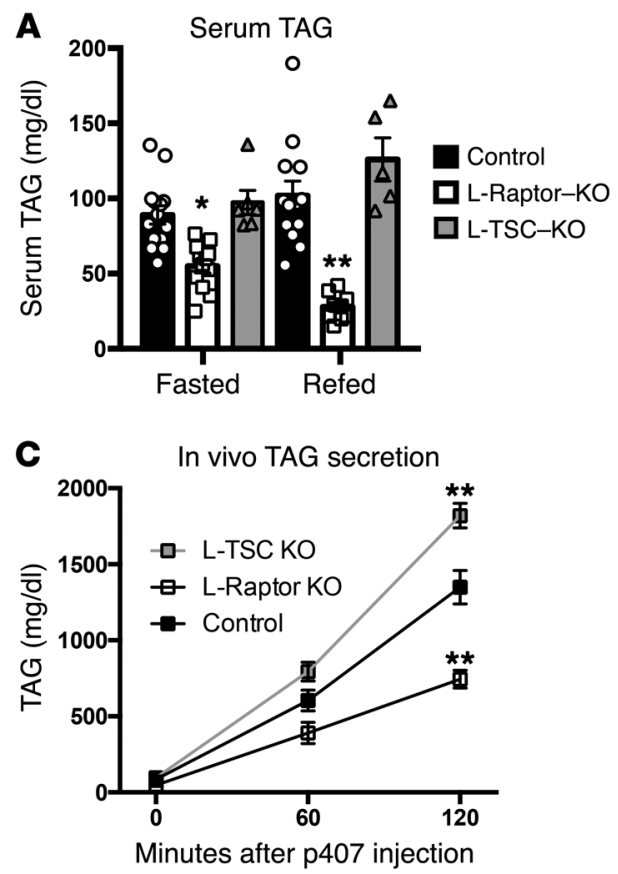

B
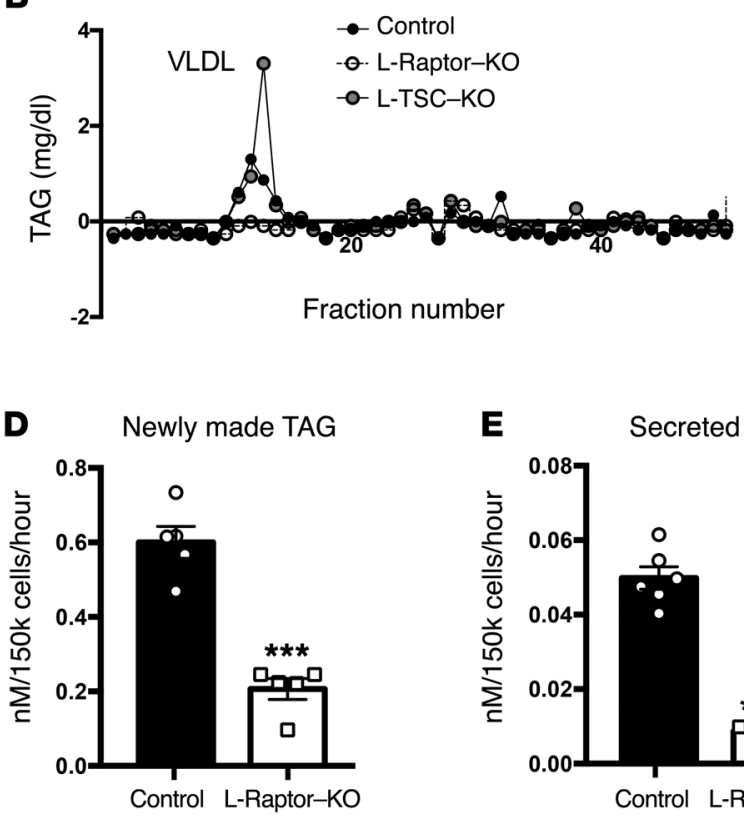
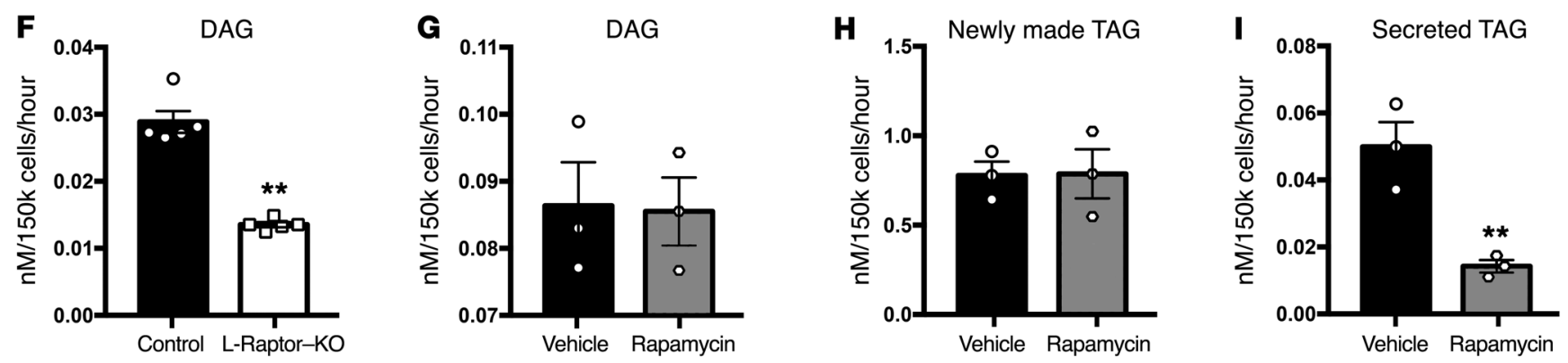

Figure 3. mTORC1 cell autonomously regulates TAG secretion. Six- to ten-week-old Raptor ${ }^{f / f l}$ and TSc1 ${ }^{f l / f l}$ animals were injected with either AAV-GFP (control, black) or AAV-CRE (L-Raptor-KO, white; L-TSC-KO, gray) for 2 weeks prior to sacrifice. (A) Serum TAG levels. $n=5-9$. (B) Serum was subjected to FPLC analysis, and triglyceride content was measured in each of the eluted fractions. (C) Triglyceride secretion rates were determined in fasted animals by blocking triglyceride uptake via i.p. injection of poloxamer 407 and measuring the accumulation of triglyceride in the serum over time. $n=4-6$ per group. (D-F) Six- to ten-week-old Raptor ${ }^{\text {fl/fl }}$ animals were injected with AAV-GFP (black) or AAV-Cre (white) and rested 2 weeks to allow for gene excision. Hepatocytes were isolated and metabolically labeled for 4 hours in culture with ${ }^{3} \mathrm{H}$-glycerol. Medium and cellular fractions were separated. Samples were then fractionated by TLC and compared with lipid standards. (D) Intracellular TAG. (E) Secreted TAG. $n=6$. (F) Intracellular DAG. $n=6$. C57BL/6 hepatocytes were isolated and metabolically labeled with ${ }^{3} \mathrm{H}$-glycerol in the presence (white) or absence (black) of rapamycin (10 ng/ml) for 4 hours. Medium and cellular fractions were separated. Samples were then fractionated by TLC and compared with lipid standards. (G) Intracellular DAG. $n=3$. (H) Intracellular TAG. $n=3$. (I) Secreted TAG. $n=3$. For hepatocyte studies in L-Raptor-KO or control, hepatocytes from 4 to 9 mice per group were isolated and technical replicates pooled. Data represent 4 to 9 individual mice per group. For hepatocyte studies using rapamycin, hepatocytes from 3 mice per group were isolated and technical replicates pooled. Data represent 3 individual mice per condition. ${ }^{*} P<0.05 ;{ }^{* *} P<0.01$; ${ }^{* * *} P<0.001$; ${ }^{* * * *} P<0.0001$ vs. control condition using 2-way ANOVA (A and C) or Student's $t$ test (D-I).

amer 407, which blocks TAG uptake, allowing the rate of secretion to be measured as the accumulation of TAG in blood (22). mTORC1 activation (L-TSC-KO) increased TAG secretion nearly 2-fold, whereas TAG secretion was reduced $50 \%$ in the absence of mTORC1 (L-Raptor-KO) (Figure 3C). These data strongly suggest that the increased liver TAG observed in L-Raptor-KO was due to reduction in VLDL-TAG secretion.

To confirm that the defect in VLDL-TAG secretion in the L-Raptor-KO mice was cell autonomous and not dependent on factors extrinsic to the liver such as FFAs, we incubated primary hepatocytes from ad libitum-fed control and L-Raptor-KO mice in the presence of $0.4 \mathrm{mM}$ oleic acid and measured production of cellular and secreted TAG. Hepatocytes isolated from L-Raptor-KO mice had a $60 \%-80 \%$ reduction in the incorporation of ${ }^{3} \mathrm{H}$-glycerol into cellular and secreted TAG (Figure 3, D and E). As reported previously (23), hepatocytes from L-Raptor-KO mice also displayed a diminution in diacylglycerol (DAG) synthesis (Figure 3F). Since this was a chronic experiment, it was not clear whether the reduction in DAG and TAG synthesis was the cause of the lower rate of secretion or an adaptation to accumulated intracellular TAG. To address this, we pretreated WT hepatocytes with $10 \mathrm{nM}$ rapamycin for 30 minutes and measured the synthesis of 
A
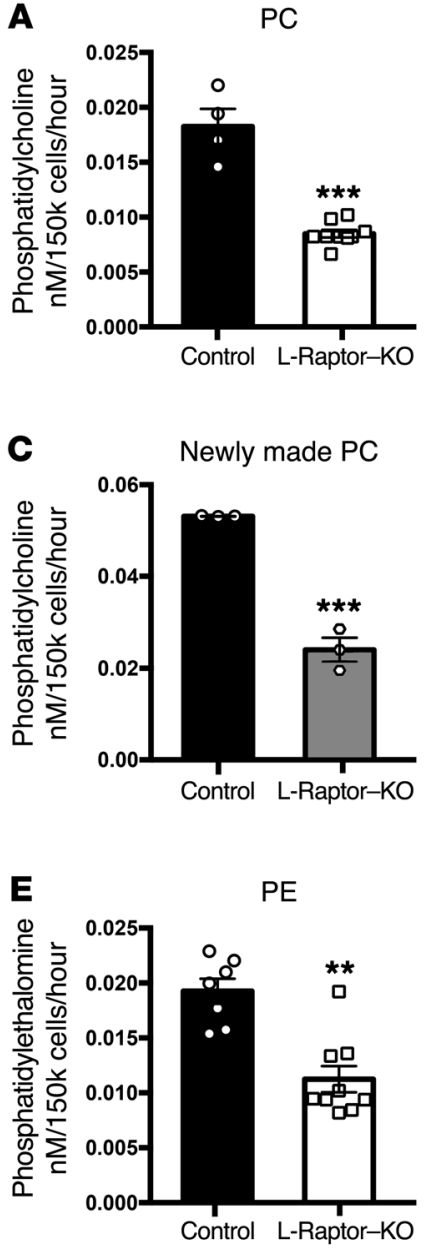

B
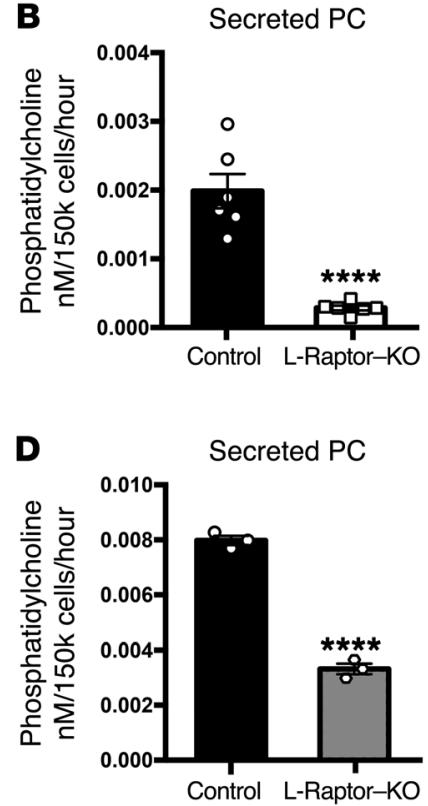

$\mathbf{F}$

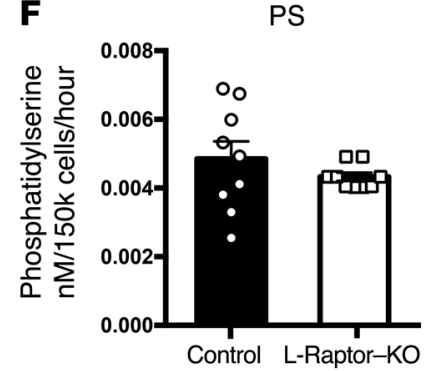

both DAG and TAG as well as secretion of the latter. As shown in Figure 3, G-I, short-term treatment with rapamycin did not affect the synthesis of DAG or TAG, while at the same time, triglyceride secretion was reduced substantially. These data are most compatible with a model in which inhibition of MTORC1 results directly in diminished TAG export from the hepatocyte, while reduced glycerolipid synthesis occurs more slowly, likely as a result of its intracellular accumulation.

MTORC1 activity is required for PC synthesis and secretion. Triglycerides are exported from the liver as VLDL particles containing lipoproteins, TAG, and cholesterol esters. Phospholipid synthesis is critical to the biogenesis and secretion of VLDL (24). PC is produced in the liver through modification of dietary choline and methionine, the Kennedy pathway, and through the methylation of phosphatidylethanolamine, the phosphatidylethanolamine $\mathrm{N}$-methyltransferase (PEMT pathway). Under normal conditions, the Kennedy pathway accounts for more than two-thirds of newly made PC in the liver (25). Based on ${ }^{3} \mathrm{H}$-glycerol incorporation, PC synthesis was reduced approximately $70 \%$ in hepatocytes from ad libitum-fed L-Raptor-KO mice (Figure 4A) while secretion of newly made PC declined over 90\% (Figure 4B). To determine whether the reduction in PC synthesis was a direct or secondary response to inhibition of mTORC1 as above, we blocked mTORC1 activity for a short period of time in isolated hepatocytes. In contrast to what occurred with TAG, 30 minutes after addition of

Figure 4. mTORC1 activity is required for PC biosynthesis and secretion. Six- to ten-week-old Raptor ${ }^{f l / f l}$ animals were injected with either AAV-GFP (control, black) or AAV-CRE (L-Raptor-KO, white) for 2 weeks prior to sacrifice. Hepatocytes were isolated and metabolically labeled for 4 hours in culture with ${ }^{3} \mathrm{H}$-glycerol. Medium and cellular fractions were separated. Samples were then fractionated by TLC and compared with lipid standards. (A) Intracellular PC. $n=4-6$. (B) Secreted PC in the medium. $n=4-6$. C57BL/6 hepatocytes were isolated and metabolically labeled with ${ }^{3} \mathrm{H}$-glycerol in the presence (white) or absence (black) of rapamycin $(10 \mathrm{ng} / \mathrm{ml})$ for 4 hours. Medium and cellular fractions were separated. Samples were then fractionated by TLC and compared with lipid standards. (C) Intracellular PC. $n=3$. (D) Secreted PC in the medium. $n=3$. (E) Intracellular PE in L-Raptor-KO. $n=6-9$. (F) Intracellular phosphatidylserine (PS) in L-Raptor-KO. $n=6-9$. For hepatocyte studies in L-Raptor-KO or control, hepatocytes from 4 to 9 mice per group were isolated and technical replicates pooled. Data represent 4 to 9 individual mice per condition. For hepatocyte studies using rapamycin, hepatocytes from 3 mice per group were isolated and technical replicates pooled. Data represent 3 individual mice per condition. ${ }^{* *} P<0.01$; ${ }^{* *} P<0.001$; ${ }^{* * * *} P<0.0001$ vs. control condition using Student's $t$ test.

rapamycin, both newly synthesized intracellular (Figure 4C) and secreted (Figure 4D) PC were reduced substantially. Synthesis of phosphatidylethanolamine, which can be made by either the Kennedy or the cytidine diphosphate-DAG (CDP-DAG) pathway, was decreased by nearly half (Figure $4 \mathrm{E}$ ) in hepatocytes isolated from ad libitum-fed L-Raptor-KO mice, while biosynthesis of phosphatidylserine, which is generated exclusively by the CDPDAG pathway, was unaffected (Figure $4 \mathrm{~F}$ ). These data suggest that mTORC1 regulates the Kennedy pathway for PC synthesis and raise the possibility that it is through this route that mTORC1 controls TAG secretion.

mTORC1 regulates CCTa protein levels posttranscriptionally. As a result of the significant defect in PC biosynthesis, we turned our efforts toward identifying the critical biochemical step that is controlled by mTORC1. Under normal conditions, the rate-limiting enzyme for PC synthesis is phosphocholine cytidylyltransferase $\alpha(C C T \alpha)$ (Figure $5 \mathrm{~A})$; inactivation of this protein genetically in mice or humans is associated with reduced hepatic triglyceride secretion and hepatosteatosis $(26,27)$. To assess the flux through $\mathrm{CCT} \alpha$, we incubated hepatocytes from ad libitum-fed mice in the presence of ${ }^{14} \mathrm{C}$-choline chloride and measured the amount of radioactivity in the product of this enzyme, CDP-choline. Incorporation of ${ }^{14} \mathrm{C}$-choline into $\mathrm{CDP}$-choline was reduced significantly in the absence of raptor (Figure 5B). Because mTORC1 is known to affect pyrimidine synthesis (28) and potentially the levels of cytidinetriphosphate (CTP), which is a substrate of CCT $\alpha$, we measured the activity of CCT $\alpha$ in cell lysates supplemented with exogenous p-choline and CTP. Under these conditions, CCT $\alpha$ activity was reduced in lysates from L-Raptor-KO livers (Figure 5C). While CCT $\alpha$ mRNA levels were equivalent between both genotypes (Figure 5D), protein levels were diminished in liver from the L-RaptorKO mice to an extent commensurate with the reduction in enzyme activity, suggesting regulation of CCT $\alpha$ stability or translation by mTORC1 at a posttranscriptional site (Figure 5E). See complete unedited blots in the Supplemental material.

Restoration of PC synthesis normalizes hepatic triglycerides and VLDL-TAG secretion in L-Raptor-KO. CCT $\alpha$-deficient animals have reduced VLDL-TAG secretion that can be restored by paren- 
A
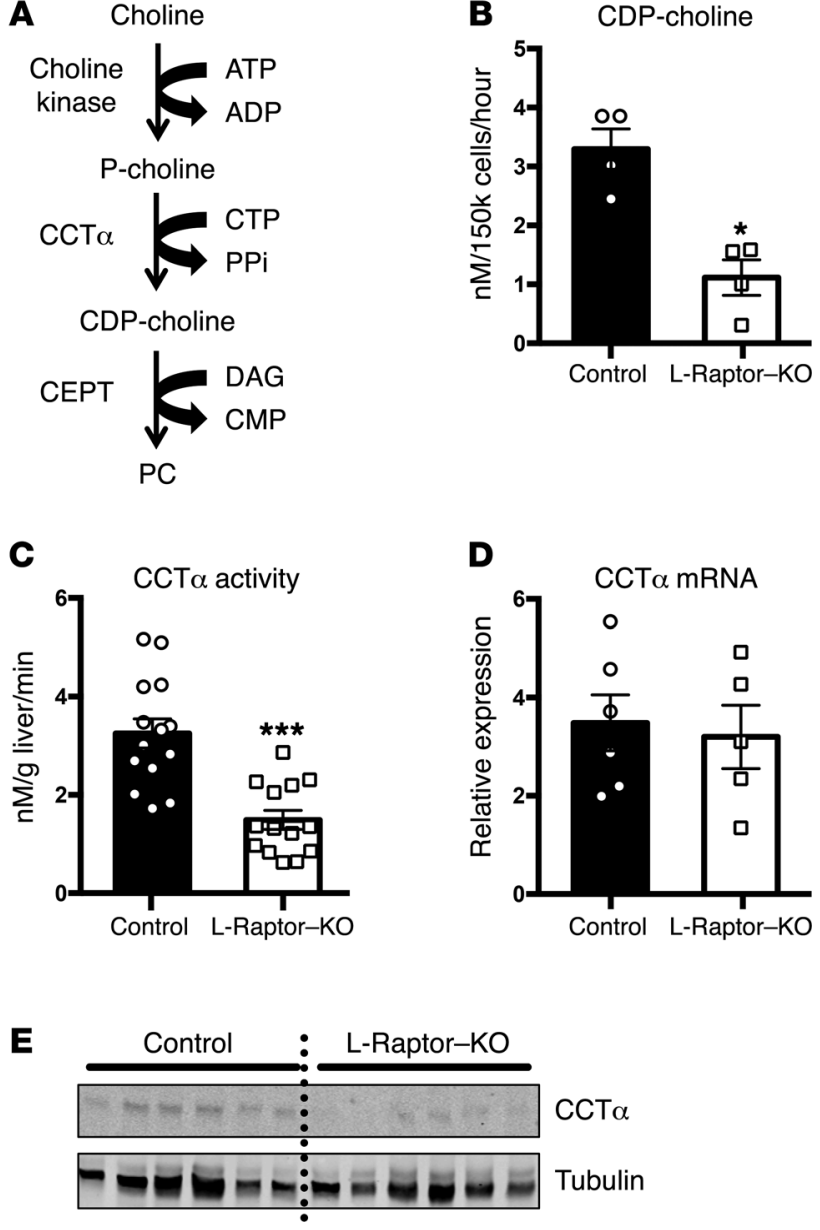

teral administration of exogenous CDP-choline (21). We reasoned that if mTORC1 regulates TAG secretion by promoting PC biosynthesis, restoration of PC synthesis would rescue the TAG secretion defect induced by loss of raptor. We therefore treated L-RaptorKO mice daily with $150 \mathrm{mg} / \mathrm{kg}$ CDP-choline for 2 weeks and measured in vivo triglyceride secretion by injecting fasted mice with poloxamer 407, a detergent that blocks lipoprotein lipase (LPL) activity (22). CDP-choline administration for 2 weeks was sufficient to rescue the rates of TAG secretion in L-Raptor-KO to a level indistinguishable from WT rates (Figure 6A). This correlated with an increase in fasting serum triglycerides in L-Raptor-KO mice (Figure 6B), and this was accompanied by a return to fasted WT levels of liver TAG (Figure 6C). Finally, we measured PC synthesis and secretion (Figure 6, D and E) in primary hepatocytes isolated from ad libitum-fed L-Raptor-KO and control mice that were incubated in the presence of CDP-choline $(5 \mu \mathrm{g} / \mathrm{ml})$. Metabolic labeling with ${ }^{3} \mathrm{H}$-glycerol confirmed that CDP-choline administration led to a significant improvement in secreted TAG in hepatocytes (Figure 6D) without affecting the rates of intracellular TAG synthesis (Figure 6E). In addition, administration of CDP-choline normalized both secreted PC (Figure 6F) and PC synthesis (Figure 6G) in the absence of mTORC1. These data support the hypothesis that loss of mTORC1 activity diminishes PC synthesis through a reduction in the generation of CDP-choline and that this leads to a decrease in VLDL-TAG secretion.
Figure 5. CCT $\alpha$ is regulated posttranscriptionally by mTORC1 activity. Six- to ten-week-old Raptor ${ }^{f l / f l}$ animals were injected with either AAV-GFP (control, black) or AAV-CRE (L-Raptor-KO, white) for 2 weeks prior to sacrifice. (A) Schematic of the Kennedy pathway. (B) Generation of CDP-choline was measured following incubation with ${ }^{14} \mathrm{C}$-choline chloride for 4 hours. $n=4$. (C) CCT $\alpha$ activity was measured in hepatocyte lysates in the presence of excess CTP and ${ }^{14} \mathrm{C}$-phosphocholine. $n=12-13$. (D) CCT $\alpha$ mRNA was interrogated by quantitative RT-PCR. $n=5-6$. (E) CCT $\alpha$ protein was measured by Western blot. Data are representative of 6 animals. ${ }^{*} P<0.05$; ${ }^{* *} P<0.001$ vs. control using Student's $t$ test.

\section{Discussion}

The initial impetus for these studies was to understand a longrecognized physiologic paradox, i.e., how does the liver maintain VLDL-TAG secretion when FFAs, the major precursor and determinant of TAG synthesis, are reduced? In exploring the topic, we uncovered a previously unrecognized pathway by which activation of mTORC1 promotes PC synthesis and, consequently, VLDL-TAG secretion to regulate hepatic lipid stores. Using both genetic manipulation and specific chemical inhibitors, we found that mTORC1 regulates the rate-limiting enzyme involved in PC synthesis, CCT $\alpha$. Indeed, as the defect in triglyceride secretion of L-Raptor-KO phenocopies CCT $\alpha$ deletion in the liver (21), administration of the metabolite CDP-choline was able to rescue the secretion defects and hepatosteatosis that result from either CCT $\alpha$ or mTORC1 deletion in liver.

These data indicate that mTORC1 is a major regulator of PC synthesis and VLDL-TAG secretion in addition to its well-characterized roles in de novo lipogenesis and $\beta$-oxidation $(15,17)$. This important metabolic function of mTORC1 is essential for the control of PC biosynthesis, for VLDL-TAG secretion, and for systemic lipid homeostasis; therefore, it is likely that mTORC1 regulation ensures appropriate lipid delivery to extrahepatic tissues for utilization and storage. mTORC1 positively regulates the level of CCT $\alpha$ protein and, consequently, its activity, though the precise mechanism remains unknown. mTORC1 is a master regulator of protein synthesis by well-established translational and posttranslational mechanisms (29). Notably, CCT $\alpha$ activity and stability are regulated by multiple phosphorylation events within its carboxy terminus (30). A phospho-proteomic screen of fasted and refed mouse livers has identified several feeding-sensitive phosphorylation sites in the carboxy terminus of CCT $\alpha$, including S315, S333, and S347 (31). Additionally, Robitaille and colleagues performed stable-isotope-labeling experiments in WT or Raptor-KO mouse embryonic fibroblasts (MEFs) and reported several phosphorylation sites on the carboxy terminus of CCT $\alpha$, including S315 and S347, though it is not clear whether the enzyme is a direct substrate (32). Therefore, our current hypothesis is that mTORC1 regulates CCT $\alpha$ activity by controlling the phosphorylation status of CCT $\alpha$. The functional significance of these sites in mediating mTORC1's control of PC biosynthesis remains to be determined.

$\mathrm{CCT} \alpha$ is an important mediator of triglyceride secretion in humans, and its inactivation leads to reduced VLDL secretion and hepatosteatosis $(21,24,26,27)$. Recent studies have tied polymorphisms in CCT $\alpha$ to inherited nonalcoholic fatty liver disease (NAFLD) (27), but it is unlikely that genetic variation of the CCT $\alpha$ gene contributes to the more common type of NAFLD, which affects nearly 1 in 3 Americans (33). Nonetheless, these data raise 
A
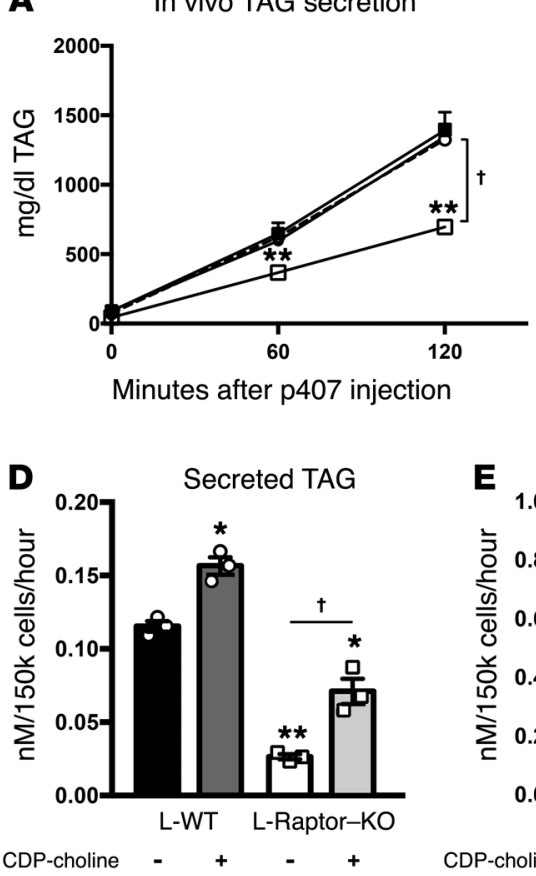
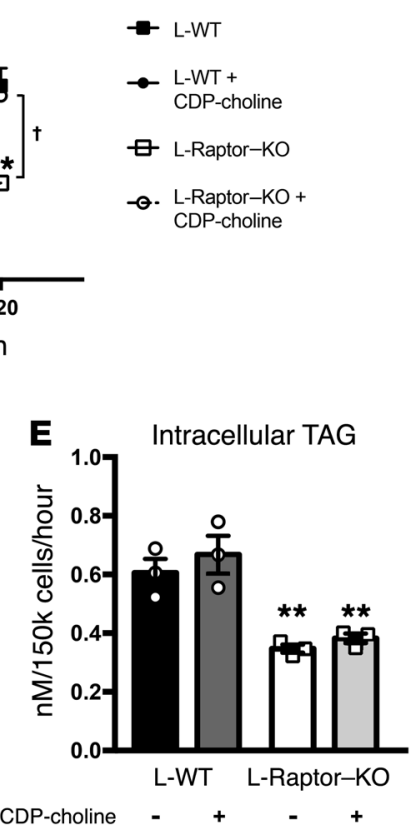

B
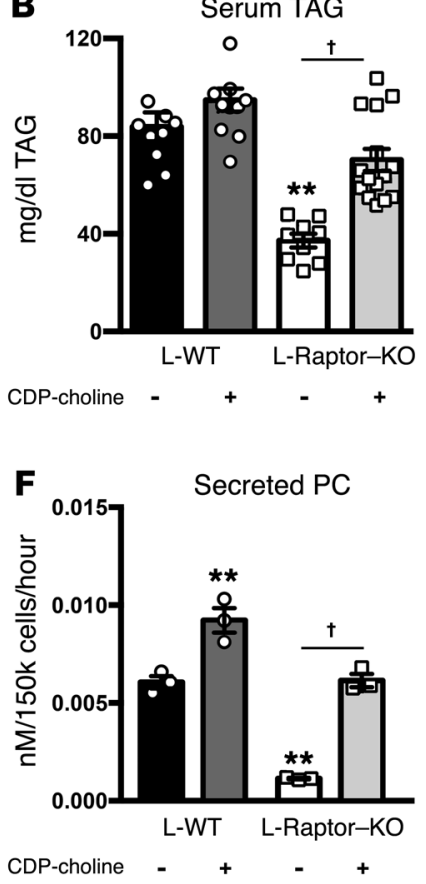
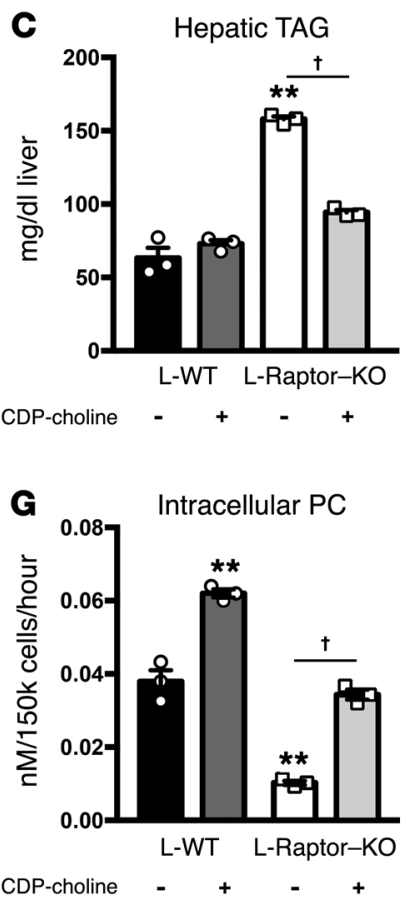

Figure 6. Restoration of PC synthesis in the absence of mTORC1 is sufficient to regulate TAG secretion in vivo. Six- to ten-week-old Raptor ${ }^{f l f l}$ animals were injected with either AAV-GFP (control, black) or AAV-CRE (L-Raptor-KO, white) and injected daily with saline (solid) or 150 mg/kg CDP-choline for 2 weeks. (A) Triglyceride secretion rates were determined in fasted animals by blocking triglyceride uptake via i.p. injection of poloxamer 407 and measuring the accumulation of triglyceride in the serum over time. $n=9-10$. (B) Fasting serum triglyceride levels were measured. $n=9-10$. (C) Fasting hepatic triglyceride levels were measured. $n=3$. Isolated hepatocytes from treatment groups were cultured with ${ }^{3} \mathrm{H}$-glycerol \pm CDP-choline (150 mg/kg) (D-G). (D) Secreted TAG was measured in the medium. $n=3$. (E) Intracellular TAG was measured. $n=3$. (F) Newly made secreted PC was measured in the medium. $n=3$. (G) Intracellular newly made PC was measured. $n=3$. For hepatocyte studies, hepatocytes from 3 mice were isolated and technical replicates pooled. Data represent 3 individual mice per condition. ${ }^{*} P<0.05 ;{ }^{* *} P<0.01$ vs. control using 1-way ANOVA. ${ }^{\dagger} P<0.01$ using 1 -way ANOVA.

the idea that decreased triglyceride secretion, possibly by a defect in PC synthesis, could contribute to the syndrome of hepatosteatosis.

Previous work has implicated hepatic insulin signaling in the progression of VLDL-TAG secretion in mouse models of metabolic disease (10). Knockdown of the hepatic insulin receptor reduces VLDL-TAG secretion while also decreasing LDL receptor (LDLR) protein levels (10). These data are consistent with studies performed in the liver insulin receptor-KO (LIRKO) mouse, which exhibits reduction in VLDL-TAG secretion and LDLR expression, yet exhibits increases in apoB export (8). This effect of insulin on LDLR protein expression is mediated in part by mTORC1 activity (34). In addition, mTORC1 activity represses sortillin 1 , which in addition to apoB metabolism, contributes to VLDL-TAG export (35). In this study, we identified another major role of mTORC1 in the regulation of lipid export and neutral lipid content in liver. Here, we demonstrate an essential role of PC biosynthesis and CCT $\alpha$ stability downstream of mTORC1 in the control of lipid homeostasis and suggest that the dominant mechanism by which mTORC1 controls VLDL-TAG secretion is through PC biosynthesis.

Changes in serum triglycerides have long been observed in rapamycin-treated humans (36). Unlike liver-specific inactivation of mTORC1 in mice, rapamycin treatment in patients and animals leads to an increase in serum triglycerides. It is not known whether these changes represent augmented secretion from liver or intestines or reduced uptake of triglycerides into the adipose tissue, as rapamycin has diverse effects in many cell types. Our study suggests that these differences are likely due to reduction in clearance of triglyceride, since hepatic inactivation of mTORC1 results in decreased serum TAG. Increased secretion is unlikely, as chronic rapamycin treatment affects mTORC1 and mTORC2 complexes, the latter leading to a decrease in Akt activation (37). This question of the tissue responsible for altered serum lipids upon systemic rapamycin treatment and which mTORC complex is involved remains largely unanswered.

In summary, we have elucidated the signaling mechanisms responsible for augmented postprandial TAG secretion in the face of reduced circulating FFA. Using primarily genetic loss-offunction experiments, we demonstrate that mTORC1 activity is required for secretion and that activation of $\mathrm{mTORC} 1$ is sufficient to increase hepatic VLDL-TAG secretion by regulating the activity of CCT $\alpha$, the rate-limiting step to PC synthesis. These data highlight an unexpected role of $\mathrm{mTORC} 1$ in the regulation of hepatic lipid homeostasis and have important implications for the systemic regulation of triglyceride homeostasis.

\section{Methods}

Animals. Raptor ${ }^{f / f l}, T s c 1^{f / / f l}$ (17), and $A k t 2^{f / f l}$ (15) mice were backcrossed to the C57BL/6 background, housed, and bred under specific pathogen-free conditions in facilities at the University of Pennsylvania. For acute excision of liver-specific genes, mice were injected with adeno- 
associated virus (Vector Core, University of Pennsylvania) containing a liver-specific promoter (TBG) at a dosage of $1.0 \times 10^{11}$ genome copies at 6 to 10 weeks of age and maintained on normal chow for another 2 weeks before analysis. Control animals consist of pools of the appropriate floxed mice (Raptor ${ }^{f / f l}, T s c 1^{f / f l}$, or $A k t 2^{f / f l} /$ Raptor $r^{f / f l}$ ) that had been injected with AAV-GFP. All experiments were performed in male mice.

Metabolic measurements. Blood glucose and hepatic and serum TAG levels were measured as described before (38). For experiments involving fasting, mice were deprived of food overnight for 16 hours. For refeeding experiments, mice were overnight fasted and refed normal chow for 4 hours before blood and liver samples were taken during the morning.

Cell culture. Hepatocytes were isolated as previously described from ad libitum-fed male mice (39). Twelve-well plates were coated with collagen and hepatocytes allowed to attach in M199 media containing 10\% FBS and $100 \mathrm{nM}$ insulin. Once attached, medium was changed to M199 medium supplemented with $0.1 \% \mathrm{BSA}, 100 \mathrm{nM}$ insulin, and $0.4 \mathrm{mM}$ oleic acid. ${ }^{3} \mathrm{H}$-glycerol $(0.1 \mu \mathrm{Ci} / \mathrm{ml}),{ }^{14} \mathrm{C}$-choline chloride $(0.1 \mu \mathrm{Ci} / \mathrm{ml})$, and/or rapamycin $(10 \mathrm{ng} / \mathrm{ml})$ were added to cultures as indicated. Cells and medium were harvested 4 hours after the addition of radioactive tracers (see below) and for TLC visualization and quantification.

Thin-layer chromatography. Primary hepatocytes were cultured and radiolabelled tracers added as indicated above. Lipid and aqueous layers were separated by a modified Bligh-Dyer method (40). Cell lysates were mixed with $\mathrm{CHCL}_{3}: \mathrm{CH}_{3} \mathrm{OH} ; 2: 1$, v:v to separate lipid and aquoes layers. Mixture was then washed two times with $\mathrm{CHCL}_{3}: \mathrm{CH}_{3} \mathrm{OH}: \mathrm{H}_{2} \mathrm{O}(3: 48: 47)+0.02 \% \mathrm{CaCl}$, and the aqueous layer was removed after each wash. Neutral lipids were separated using C6H12: $\left(\mathrm{C}_{2} \mathrm{H}_{5}\right)_{2} \mathrm{O}: \mathrm{C}_{2} \mathrm{H}_{4} \mathrm{O}_{2}$ (170:30:1; v:v) followed by $\mathrm{CHCL}_{3}: \mathrm{CH}_{3} \mathrm{OH}$ : $\mathrm{H}_{2} \mathrm{O}: \mathrm{C}_{2} \mathrm{H}_{4} \mathrm{O}_{2}$ (100:104:12:2; v:v) to mobilize charged lipids on Silica Gel $\mathrm{G}$, which allow for resolution of neutral lipids and phospholipids. Aqueous fractions were separated using $\mathrm{CH}_{3} \mathrm{OH}: 6 \% \mathrm{NaCl}$ : $\mathrm{NH}_{3} \mathrm{OH}(10: 10: 1 ; \mathrm{v}: \mathrm{v})$ on silica gel G-channeled plates. All unknowns were confirmed by comigration of purified standards. Lipids were detected with iodine. Plates were then scraped and radioactivity counted using a liquid scintillator. Concentrations of unknowns were calculated using the specific activity of the tracers.

In vivo triglyceride secretion. Triglyceride secretion was measured in vivo by i.p. injection of poloxamer 407 and measurement of accumulated triglycerides in the serum, as described previously (22).

Fractionation FPLC. Plasma lipoprotein distribution in VLDL, LDL, and HDL regions was performed using fast protein LC (FPLC) fractionation on a Superose $610 / 300$ column (GE Healthcare). Equal volumes $(150 \mu \mathrm{l})$ of pooled plasma samples from each group (5 mice per group) were subjected to FPLC fractionation using FPLC buffer (10 mM TRIS, $140 \mathrm{mM} \mathrm{NaCl}, 0.01 \%$ sodium azide, 0.01\% EDTA balanced to $\mathrm{pH}$ 7.4). TAG concentrations in each fraction were measured using commercially available colorimetric reagents (Wako) as described earlier (41).

CCT $\alpha$ activity. CCT $\alpha$ activity was determined as described previously (42). Cell protein lysates $(300 \mu \mathrm{g})$ were assayed in a reaction mixture containing $100 \mathrm{mM}$ bis-Tris-HCl, pH 6.5, 2 mM CTP, $20 \mathrm{mM} \mathrm{MgCl}_{2}, 2.5$ $\mathrm{mM}$ phospho(methyl ${ }^{14} \mathrm{C}$ ) choline, $50 \mu \mathrm{M}$ oleic acid, and $50 \mu \mathrm{M}$ PC in a final volume of $40 \mu \mathrm{l}$. Reactions were incubated for 20 minutes at $37^{\circ} \mathrm{C}$ and were terminated by adding $5 \mu \mathrm{l}$ of $0.5 \mathrm{mM}$ EDTA. CDP $\left({ }^{14} \mathrm{C}\right)$ choline formation was quantified by spotting $30 \mu \mathrm{l}$ of reaction on a Silica Gel G thin-layer plate that was developed with methanol/0.6\% NaCl/ $\mathrm{NH}_{3} \mathrm{OH}$ $(10 / 10 / 1, v / v)$. The amount of radioactivity on the plate was visualized under UV light, scraped, and quantified in a liquid scintillator.

Immunoblots. Protein lysates were prepared from frozen livers or hepatocytes in a modified RIPA buffer as described previously (14). The following antibodies were used for immunoblotting: p-Akt (cata$\log$ 4060), Akt (catalog 2964), p-S6 (catalog 2215), S6 (catalog 2217), Tsc1 (catalog 6935), Raptor (catalog 2280), and CCT (catalog 6931). All antibodies were from Cell Signaling.

Statistics. All data are presented as mean \pm SEM. Statistical analysis was performed using 1-way ANOVA when more than 2 groups were compared, 2-way ANOVA when 2 conditions were involved, and unpaired 2-tailed Student's $t$ test when only 2 groups of data were concerned. $P<0.05$ was considered statistically significant.

Study approval. All animal studies were approved by the IACUC board of the University of Pennsylvania, Philadelphia, PA, USA.

\section{Author contributions}

WJQ conceived the hypothesis, designed and performed the experiments, analyzed the data, and prepared the manuscript. MW contributed to discussion, performed experiments, and analyzed data. SVS performed experiments and contributed to discussion and preparation of the manuscript. RGG performed experiments. DJR contributed to discussion and edited the manuscript. MJB conceived the hypothesis, codirected the project, and prepared the manuscript. PMT conceived the hypothesis, designed and performed the experiments, analyzed the data, codirected the project, and prepared the manuscript. All authors approved the final version of the manuscript.

\section{Acknowledgments}

The Viral Vector Core of the University of Pennsylvania, supported by the Diabetes Research Center (NIH DK19525), provided viruses. This work was supported by NIH grant R01 DK056886 (to MJB), K01 DK111715 (to PMT), the Samuel Chiaffa Memorial Fund (to PMT), Institute for Diabetes, Obesity, and Metabolism of the University of Pennsylvania start up funds (to PMT), and T32 DK007314 (to WJQ). SVS is supported by an American Diabetes Association postdoctoral fellowship (\#1-17-PDF-060).

Address correspondence to: Paul M. Titchenell, 3400 Civic Center Boulevard, Philadelphia, Pennsylvania 19104, USA. Phone: 215.573.1872; Email: ptitc@pennmedicine.upenn.edu. Or to: Morris J. Birnbaum, Internal Medicine, Pfizer Inc., 1 Portland Street, Cambridge, Massachusetts 02139, USA. Phone: 617.512.6227; Email: morris.birnbaum@pfizer.com.

M. Wan's present address is: Agios Pharmaceuticals, Cambridge, Massachusetts, USA.
1. Adamovich Y, Aviram R, Asher G. The emerging roles of lipids in circadian control. Biochim Biophys Acta. 2015;1851(8):1017-1025.

2. Browning JD, Baxter J, Satapati S, Burgess SC.
The effect of short-term fasting on liver and skeletal muscle lipid, glucose, and energy metabolism in healthy women and men. J Lipid Res. 2012;53(3):577-586
3. Lambert JE, Parks EJ. Postprandial metabolism of meal triglyceride in humans. Biochim Biophys Acta. 2012;1821(5):721-726.

4. Tiwari S, Siddiqi SA. Intracellular trafficking and 
secretion of VLDL. Arterioscler Thromb Vasc Biol. 2012;32(5):1079-1086.

5. Sparks JD, Sparks CE, Adeli K. Selective hepatic insulin resistance, VLDL overproduction, and hypertriglyceridemia. Arterioscler Thromb Vasc Biol. 2012;32(9):2104-2112.

6. Haas ME, Attie AD, Biddinger SB. The regulation of ApoB metabolism by insulin. Trends Endocrinol Metab. 2013;24(8):391-397.

7. Steiner G, Haynes FJ, Yoshino G, Vranic M. Hyperinsulinemia and in vivo very-low-density lipoprotein-triglyceride kinetics. Am J Physiol. 1984;246(2 pt 1):E187-E192.

8. Biddinger SB, et al. Hepatic insulin resistance is sufficient to produce dyslipidemia and susceptibility to atherosclerosis. Cell Metab. 2008;7(2):125-134.

9. Haas JT, et al. Hepatic insulin signaling is required for obesity-dependent expression of SREBP-1c mRNA but not for feeding-dependent expression. Cell Metab. 2012;15(6):873-884.

10. Han S, et al. Hepatic insulin signaling regulates VLDL secretion and atherogenesis in mice. JClin Invest. 2009;119(4):1029-1041.

11. Duerden JM, Gibbons GF. Storage, mobilization and secretion of cytosolic triacylglycerol in hepatocyte cultures. The role of insulin. Biochem J.1990;272(3):583-587.

12. Salhanick AI, Schwartz SI, Amatruda JM. Insulin inhibits apolipoprotein B secretion in isolated human hepatocytes. Metab Clin Exp. 1991;40(3):275-279.

13. Laplante M, Sabatini DM. mTOR signaling in growth control and disease. Cell. 2012;149(2):274-293.

14. Titchenell PM, et al. Direct hepatocyte insulin signaling is required for lipogenesis but is dispensable for the suppression of glucose production. Cell Metab. 2016;23(6):1154-1166.

15. Wan M, et al. Postprandial hepatic lipid metabolism requires signaling through Akt2 independent of the transcription factors FoxA2, FoxO1, and SREBP1c. Cell Metab. 2011;14(4):516-527.

16. Yecies JL, et al. Akt stimulates hepatic SREBP1c and lipogenesis through parallel mTORC1dependent and independent pathways. Cell Metab. 2011;14(1):21-32.

17. Sengupta S, Peterson TR, Laplante M, Oh S, Saba- tini DM. mTORC1 controls fasting-induced ketogenesis and its modulation by ageing. Nature. 2010;468(7327):1100-1104.

18. Leavens KF, Birnbaum MJ. Insulin signaling to hepatic lipid metabolism in health and disease. Crit Rev Biochem Mol Biol. 2011;46(3):200-215.

19. Huang J, Manning BD. A complex interplay between Akt, TSC2 and the two mTOR complexes. Biochem Soc Trans. 2009;37(pt 1):217-222.

20. Kawano Y, Cohen DE. Mechanisms of hepatic triglyceride accumulation in non-alcoholic fatty liver disease. J Gastroenterol. 2013;48(4):434-441.

21. Niebergall LJ, Jacobs RL, Chaba T, Vance DE. Phosphatidylcholine protects against steatosis in mice but not non-alcoholic steatohepatitis. Biochim Biophys Acta. 2011;1811(12):1177-1185.

22. Millar JS, Cromley DA, McCoy MG, Rader DJ, Billheimer JT. Determining hepatic triglyceride production in mice: comparison of poloxamer 407 with Triton WR-1339. J Lipid Res. 2005;46(9):2023-2028.

23. Peterson TR, et al. mTOR complex 1 regulates lipin 1 localization to control the SREBP pathway. Cell. 2011;146(3):408-420.

24. Yao ZM, Vance DE. The active synthesis of phosphatidylcholine is required for very low density lipoprotein secretion from rat hepatocytes. J Biol Chem. 1988;263(6):2998-3004.

25. Sundler R, Akesson B. Regulation of phospholipid biosynthesis in isolated rat hepatocytes. Effect of different substrates. J Biol Chem. 1975;250(9):3359-3367.

26. Vance DE, Vance JE. Physiological consequences of disruption of mammalian phospholipid biosynthetic genes. JLipid Res. 2009; 50(suppl):S132-S137.

27. Payne F, et al. Mutations disrupting the Kennedy phosphatidylcholine pathway in humans with congenital lipodystrophy and fatty liver disease. Proc Natl Acad Sci U S A. 2014;111(24):8901-8906.

28. Ben-Sahra I, Howell JJ, Asara JM, Manning BD. Stimulation of de novo pyrimidine synthesis by growth signaling through mTOR and S6K1. Science. 2013;339(6125):1323-1328.

29. Dibble CC, Manning BD. Signal integration by mTORC1 coordinates nutrient input with biosynthetic output. Nat Cell Biol. 2013;15(6):555-564.
30. Yang W, Jackowski S. Lipid activation of CTP:phosphocholine cytidylyltransferase is regulated by the phosphorylated carboxyl-terminal domain. J Biol Chem. 1995;270(28):16503-16506.

31. Wilson-Grady JT, Haas W, Gygi SP. Quantitative comparison of the fasted and re-fed mouse liver phosphoproteomes using lower $\mathrm{pH}$ reductive dimethylation. Methods. 2013;61(3):277-286.

32. Robitaille AM, et al. Quantitative phosphoproteomics reveal mTORC1 activates de novo pyrimidine synthesis. Science. 2013;339(6125):1320-1323.

33. Cohen JC, Horton JD, Hobbs HH. Human fatty liver disease: old questions and new insights. Science. 2011;332(6037):1519-1523.

34. Ai D, et al. Regulation of hepatic LDL receptors by MTORC1 and PCSK9 in mice. JClin Invest. 2012;122(4):1262-1270.

35. Ai D, et al. Activation of ER stress and mTORC1 suppresses hepatic sortilin-1 levels in obese mice. J Clin Invest. 2012;122(5):1677-1687.

36. Morrisett JD, et al. Effects of sirolimus on plasma lipids, lipoprotein levels, and fatty acid metabolism in renal transplant patients. J Lipid Res. 2002;43(8):1170-1180.

37. Lamming DW, et al. Rapamycin-induced insulin resistance is mediated by mTORC2 loss and uncoupled from longevity. Science. 2012;335(6076):1638-1643.

38. Leavens KF, Easton RM, Shulman GI, Previs SF, Birnbaum MJ. Akt2 is required for hepatic lipid accumulation in models of insulin resistance. Cell Metab. 2009;10(5):405-418.

39. Titchenell PM, Chu Q, Monks BR, Birnbaum MJ. Hepatic insulin signalling is dispensable for suppression of glucose output by insulin in vivo. Nat Commun. 2015;6:7078.

40. Bligh EG, Dyer WJ. A rapid method of total lipid extraction and purification. Can J Biochem Physiol. 1959;37(8):911-917.

41. Shewale SV, et al. Botanical oils enriched in n- 6 and n-3 FADS2 products are equally effective in preventing atherosclerosis and fatty liver. J Lipid Res. 2015;56(6):1191-1205.

42. Lykidis A, Murti KG, Jackowski S. Cloning and characterization of a second human CTP:phosphocholine cytidylyltransferase. J Biol Chem. 1998;273(22):14022-14029. 